\title{
Nomenclature and Synonyms of the Umbilicus
}

Nomenclature: Umbilicus, belly button, navel, omphalos, outie, innie, centrepiece of the human body, and sole button

Umbilical cord known as: Lifeline, thread of life, funis and funiculus, which is derived from the Latin word used for rope or cord

Umbilicus is the common scientific and medical name, but in the common language, it is called belly button; it represents the rounded, knotty depression in the centre of the abdomen caused by the detachment of the umbilical cord.
Navel: The term omphalos in Greek or umbilicus in Latin means a 'navel' which is a conical stone that was deemed in antiquity to have marked a 'Centre of the Earth'.

Omphalos: This is the scientific and medical term used to represent the umbilicus; the origin of this name will be explained later.

Outie and innie: In humans, the umbilical scar can appear as a depression (often referred to as an innie) or as a protrusion (outie), although umbilicus could be classified into these two categories. 